\title{
EFFECT OF NUTRITIONAL STATUS ON HEALTH- RELATED QUALITY OF LIFE (HRQL) THROUGHOUT CHEMOTHERAPY: A PROSPECTIVE STUDY
}

Isis D. D. Custódio¹, Eduarda da C. Marinho1, Carlos E. Paiva1, Yara C. de P. Maia1

'Universidade Federal de Uberlândia - Uberlândia - (MG), Brazil.

This prospective study aimed to evaluate the effect of nutritional status and chemotherapy (CT) time; beginning (T0), during (T1) and after the end of the CT (T2); as well as the interaction between these variables on the domains of healthrelated quality of life (HRQL). Anthropometric data was performed including 55 women with breast cancer, and the HRQL was analyzed with the EORTC QLQ-30 and BR23 instruments, 21 days after each investigated time, always before CT. Body Mass Index (BMI) and waist circumference (WC) increased significantly ( $\mathrm{p}=0.009$ and $\mathrm{p}=0.030$, respectively), in T2 when compared to T0. The Generalized Estimated Equations (GEE) were controlled by age, the estimated marginal means was analyzed, and 95\% confidence intervals were compared pairwise by applying Sidak for multiple tests. The domains functional global, physical function and symptoms were significantly associated with BMI (equation 1 : $p=0.025, p=0.019$ and $\mathrm{p}=0.016$, respectively) and $\mathrm{WC}$ (equation $2: \mathrm{p}=0.015, \mathrm{p}=0.016$ and $\mathrm{p}=0.024$, respectively), with the CT time (equation 1: $p<0.001, p<0.001$ and $p=0.003$; equation 2 : $p<0.001, p=0.001$ and $p=0.013$, respectively), with the interaction between CT time and BMI (equation $1: p=0.032, p=0.012$ and $p=0.042$, respectively), and with CT time and WC (equation 2: $p=0.028$, $\mathrm{p}=0.035$ and $\mathrm{p}=0.024$, respectively). When assessing the post-hoc (Sidak), it was identified that the worse scores of HRQL domains were observed in T2, and these were associated with worst nutritional status. Higher BMI and WC negatively impacted the HRQL, situation worsened with the passing of CT time, which could harm the treatment and survival of these women. Actions aimed at minimizing the impairment in HRQL, such as maintaining healthy nutritional status, need to be adopted to reduce the effects of this exhausted treatment. 